\title{
Le lichen plan gingival érosif : à propos de 3 cas
}

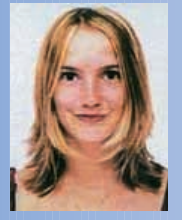

Caroline GERBER

Service d'odontologie CHU Nancy, France.

\section{Cécile LUCAS}

Service d'odontologie CHU Nancy, France.

\section{Alexandra SOURDOT}

Service d'odontologie CHU Nancy, France.

\section{Stéphane KIRCHNER}

Cabinet de pathologie,

5 , rue Isabey,

54000 Nancy.

\section{Pierre BRAVETTI}

Maître de conférence,

Praticien hospitalier

à la faculté d'odontologie de Nancy,

Doyen de la faculté d'odontologie,

Responsable du département

de chirurgie et pathologie buccale,

Service d'odontologie,

CHU de Nancy.
Le lichen plan buccal est une dermatose inflammatoire chronique, généralement bénigne et dont le diagnostic repose sur un examen clinique rigoureux et l'analyse anatomopathologique.

II se caractérise par des formes cliniques variées dont le lichen plan buccal érosif qui correspond à $40 \%$ des lésions.

Cette forme particulière se distingue par une symptomatologie et des signes cliniques et histologiques particuliers. Généralement hyperalgique, ce type de lésions nécessite un traitement à base de corticoïdes locaux ou de rétinoïdes topiques.

Trois cas cliniques viendront illustrer notre propos, nous permettant d'aborder l'association entre le lichen plan buccal et certaines pathologies systémiques, ainsi que la notion de gingivite érosive chronique. 


\section{Introduction}

Le lichen plan est une dermatose inflammatoire chronique généralement bénigne de la peau et des muqueuses malpighiennes, caractérisée par un trouble de la kératinisation, et dont les aspects cliniques sont polymorphes. Le ratio femme/homme est de $2,3 / 1$. Son incidence varie, selon les études, de 0,1 à $4 \%$ les sujets de la quatrième et de la cinquième décennie étant les plus touchés $[1,4]$.

Si la physiopathologie du lichen plan n'est pas tout à fait élucidée, I'hypothèse d'une prédisposition génétique et d'une réaction immunitaire à lymphotoxicité dirigée contre la membrane basale a été retenue. Les irritants chroniques tels que la consommation de tabac, d'alcool et de bétel, ou un traumatisme mécanique chronique sont considérés comme des cofacteurs aggravants [5, 7]. L'importance du terrain psychique (stress, dépression, anxiété) est également évoquée $[9,10]$. L'association du lichen plan avec I'hépatite $C$ est soumise à controverse [15, 16, 17].

Soulignons enfin l'existence de lésions dites lichénoïdes (dites à tort «lichen plan buccal induit»), qui sont cliniquement et histologiquement identiques aux lésions du lichen plan buccal. Elles sont causées par des traitements médicamenteux systémiques (antipaludéens de synthèse, antidépresseurs, sels d'or, D-pénicillamine, certains antituberculeux, diurétiques, hypoglycémiants, AINS, allopurinol...), par des matériaux utilisés pour les restaurations dentaires (mercure, or, chrome, sulfate de cuivre...) $[11,12,13]$, ou par une greffe de moelle osseuse (réaction du greffon contre I'hôte) [14].

Le diagnostic du lichen plan buccal repose sur des critères cliniques et histologiques [2].
Selon l'OMS (1997), le lichen plan buccal regroupe trois formes cliniques: une forme réticulée, caractéristique et correspondant à la phase quiescente de la maladie; une forme érosive, correspondant à une poussée aiguë, et une forme atrophique, forme tardive ou état post-lichénien présentant un risque de transformation maligne accru $[2,9]$. Il existe également des formes hyperkératosiques ou bulleuses.

Le siège électif des lésions est la muqueuse jugale postéro-inférieure, avec le plus souvent des atteintes bilatérales, plus ou moins symétriques. Les autres localisations sont, par ordre décroissant: le dos de la langue, la fibromuqueuse gingivale, la fibromuqueuse palatine, la face ventrale de la langue, la muqueuse ou demi-muqueuse labiale et le plancher de bouche [6]. Les plaintes fonctionnelles sont de type brûlures, douleurs, difficultés à l'alimentation, sensations rugueuses, sécheresse buccale, douleurs gingivales [1, 3].

Des examens biologiques peuvent être demandés ; la numération formule sanguine objectivant souvent une hyperéosinophilie, l'augmentation de la vitesse de sédimentation et de la protéine $\mathrm{C}$ active soulignant quant à elles le processus inflammatoire.

Le diagnostic du lichen plan doit être confirmé par un examen anatomopathologique [8]. L'examen microscopique montre d'une part un infiltrat lymphocytaire en bande sous-épithéliale dans le derme superficiel, entraînant la lyse de certains kératinocytes basaux objectivée par des corps colloïdes ou corps de Civatte. D'autre part, l'épithélium sus-jacent est le siège d'une hyperkératose et d'une hyperacanthose avec prolongements interpapillaires effilés en dents de scie $[6,29,30]$. 
Le traitement du lichen plan buccal dépend de sa forme clinique.

La forme quiescente, asymptomatique, ne nécessite pas de traitement mais une surveillance obligatoire, tous les trois mois en moyenne. Les irritants locaux (alcoolo-tabagisme, prothèses mal adaptées, dents délabrées, électro-galvanisme, mauvaise hygiène bucco-dentaire...) doivent être supprimés. La mise en place d'un suivi psychologique peut s'avérer nécessaire [10, 19].

Le traitement des formes érosives, symptomatiques, fait appel à une corticothérapie locale (Buccobet ${ }^{\circledR}$ ) et/ou, en l'absence de résultats, à des rétinoïdes topiques (Locacid ${ }^{\circledR}$ ) $[20,22]$. Pour les formes sévères ou résistantes aux traitements locaux, la corticothérapie générale ou mixte est indiquée. En alternative est proposée

\section{Cas $n^{\circ} 1$ : Mme A.}

Une patiente, âgée de 57 ans, consulte en 2009 pour un épisode de brûlures, picotements et sensations de gonflements de la muqueuse buccale, associés à une dysphagie et des douleurs au brossage.

La patiente, outre ses antécédents médicaux de thyroïdite d'Hashimoto, d'asthme et de dermatose papuleuse des coudes (étiquetée «granulome annulaire disséminé»), rapporte une fatigue intense depuis plusieurs années. Malgré une série d'hospitalisations et de bilans suite à des vertiges, pertes de connaissance et dorsalgies, une errance diagnostique demeure concernant cette asthénie qui persiste, selon la patiente, depuis 2003.

À cette date, et pendant 3 mois, elle a présenté des récurrences hebdomadaires d'angioœdèmes cervico-faciaux (langue, paupières,
I'utilisation de rétinoïdes par voie systémique [21]. Sur les lichens plans très actifs, le recours aux immunosuppresseurs peut s'avérer utile mais avec une grande prudence.

Le lichen plan buccal érosif est une forme de lichen plan constatée en atteinte initiale dans $40 \%$ des cas. Le patient consulte car il a des difficultés à s'alimenter et à réaliser ses soins bucco-dentaires quotidiens. Cette forme buccale se caractérise par des érosions douloureuses rouge vif vernissé, l'aspect lichénien typique, réticulé, étant souvent retrouvé en filigrane en périphérie des lésions.

L'atteinte gingivale correspond à environ $40 \%$ des localisations [26, 27].

Nous développerons ici la localisation gingivale du lichen plan érosif à travers 3 cas cliniques.

lèvres...) unilatéraux, associés à un prurit. Ces symptômes rentrent dans le cadre d'une urticaire chronique dont l'étiologie est à confirmer. L'hypothèse d'œdème angio-neurotique $a$ été écartée par le dosage de l'inhibiteur de C1 estérase. La symptomatologie a régressé suite au traitement par antihistaminiques (Aerius ${ }^{\circledR}$ ) et corticoïdes (Solupred ${ }^{\circledR}$ ).

Suite aux bilans infructeux concernant son asthénie, la patiente a consulté différents spécialistes, sans amélioration durable des symptômes. Lorsque la patiente nous consulte en février 2009, une fatigue générale perdure, et de nouveaux épisodes d'œdèmes sont rapportés depuis avril 2008.

Au niveau buccal, une gingivite desquamative, diagnostiquée par un dermatologue, évolue depuis plus de 10 ans. Lors de l'interrogatoire, 
la patiente explique également qu'elle portait des couronnes métalliques depuis 1992 et qu'une allergie aux métaux a été suspectée. Un bilan allergologique a montré une sensibilisation au sulfate de nickel et au palladium, traitée par des chélateurs dans un premier temps. Puis, en 2007, les couronnes céramo-métalliques et les amalgames ont été déposés, et remplacés par des couronnes céramo-céramiques.

Suite à cela, une amélioration gingivale a été notée pendant 2 à 3 mois, ainsi que l'absence de manifestation cutanée. Cependant, 3 mois plus tard, une aggravation des symptômes accompagnant l'asthénie s'est produite (hypotension, diarrhées, douleurs, perte de poids).

À l'examen exobuccal, aucune adénopathie, tuméfaction, ni coloration des téguments n'est constatée. À l'examen endobuccal, I'hygiène de la patiente est correcte et l'adaptation cervicale des couronnes céramo-céramiques est bonne. L'examen dentaire minutieux et le bilan radiologique (radiographie panoramique dentaire et cone beam 3D) ne retrouvent aucun foyer infectieux bucco-dentaire ce qui permet d'éliminer l'hypothèse d'une urticaire chronique avec angio-œdèmes d'origine dentaire.

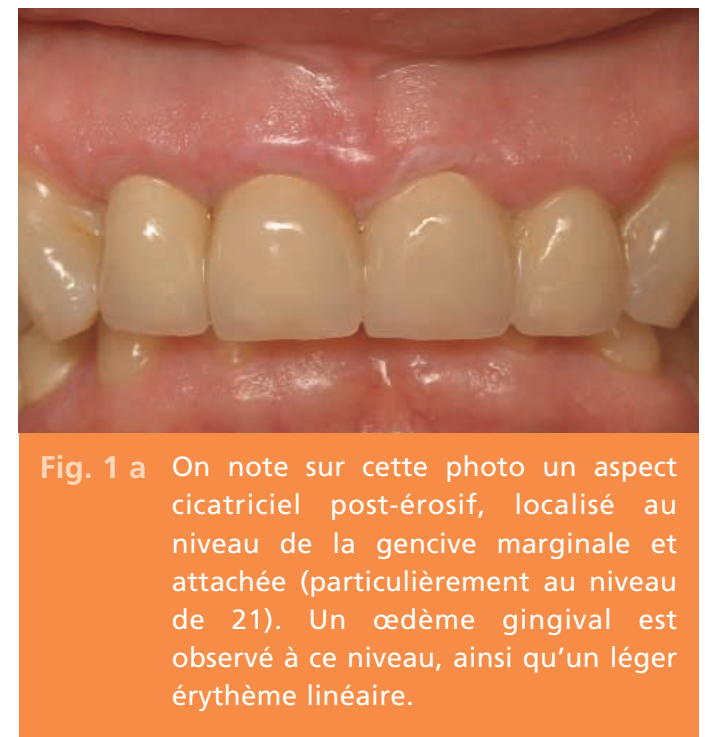

L'examen des muqueuses révèle un aspect vernissé et cicatriciel de la gencive attachée de toutes les dents (fig. 1 a et $\mathbf{1}$ b). Par endroits, la gencive prend un aspect kératosique discrètement réticulé. II existe également des zones labiles de desquamation de l'épithélium gingival au collet de certaines dents.

Afin de préciser la cause de cette gingivite desquamative, une biopsie est envisagée ; 2 fragments gingivaux sont prélevés : le premier est conservé dans du liquide de Bouin en vue d'un examen anatomopathologique, tandis que le second est réfrigéré et transporté dans de l'azote liquide pour réaliser une immunofluorescence.

L'examen histologique révèle une hyperkératose orthokératosique associant une hyperacanthose avec des crêtes épithéliales étirées en dents de scie et la présence de corps colloïdes avec un aspect liquéfié de ces assises basales. De plus, le chorion est le siège d'un infiltrat inflammatoire dense mononucléé avec une prédominance d'éléments lymphocytaires. L'immunofluorescence à la recherche d'anticorps anti-espaces intercellulaires est restée négative mais l'examen anatomopathologique a permis de conclure à un lichen plan buccal de

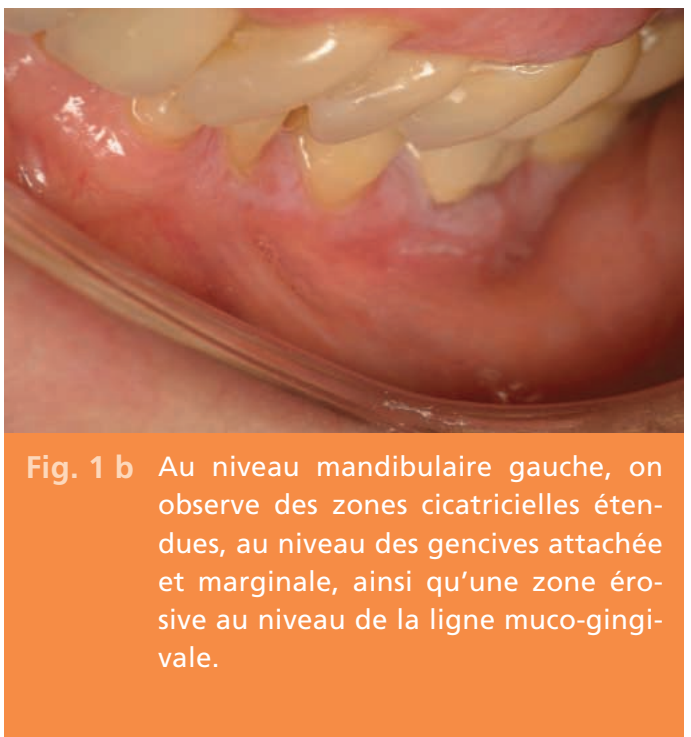


type érosif, bénin. L'hypothèse de la pemphigoïde cicatricielle avait été écartée en premier lieu, le signe de la pince effectué chez la patiente étant négatif.

La patiente a donc été traitée par une association de corticoïdes locaux (Buccobet ${ }^{\circledR}$ ) et réti-

\section{Cas no 2 : Mme F.}

Lors de la première consultation, une patiente de 54 ans nous explique qu'un diagnostic de gingivite desquamative a été posé par son chirurgien-dentiste et que la pathologie perdure depuis plusieurs mois. Ainsi, elle est adressée par le praticien odontologiste traitant car celle-ci ne constate aucune amélioration clinique, ni symptomatologique suite au traitement prescrit, à savoir des bains de bouche à base de chlorhexidine à $0,12 \%$.

Par ailleurs, la patiente présente un bon état général, sans antécédent médical excepté une

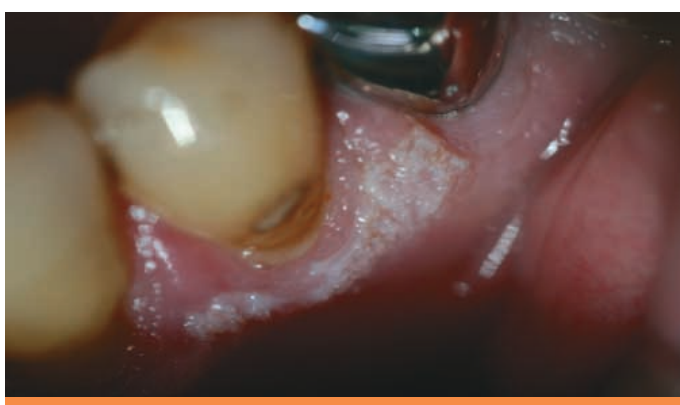

Fig. 2 Au niveau de la papille interdentaire des dents 33-34, une zone érosive érythémateuse est constatée. Plus à distance, les collets sont soulignés de plages hyperkératosiques, siégeant au niveau de la gencive attachée. noïdes topiques (Locacid $^{\circledR}$ ). Depuis, une régression de la symptomatologie buccale est constatée et l'état général de la patiente s'est amélioré. Une surveillance trimestrielle est entreprise.

sévère intoxication tabagique (45 paquetsannée environ).

L'examen clinique exobuccal ne détecte ni tuméfaction, ni adénopathie, ni lésion cutanée. L'examen de la cavité buccale révèle une hygiène bucco-dentaire moyenne, avec présence de restaurations dentaires multiples, notamment par des couronnes coulées. L'examen des muqueuses met en évidence la présence d'érosions gingivales associées à des zones hyperkératosiques au collet des dents des deux arcades (fig. 2) et sur la face interne des joues.

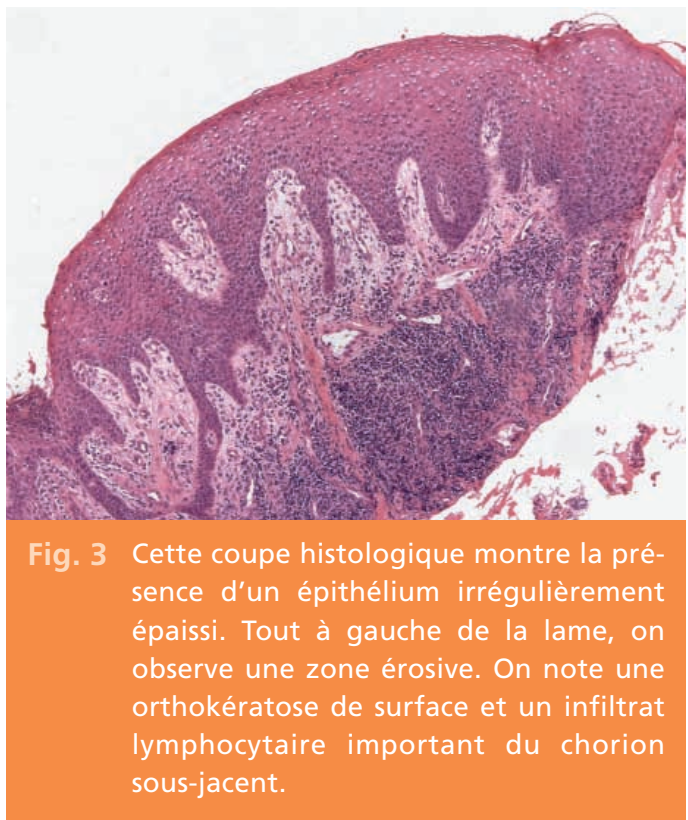


Le bilan sanguin montre une NFS normale mais une CRP augmentée prouvant l'existence d'un syndrome inflammatoire actuel ou en régression.

Une biopsie est réalisée à cheval sur une lésion, afin de préciser le diagnostic étiologique de cette gingivite desquamative. L'examen par immunofluorescence directe ne met pas en évidence de dépôts d'immunoglobuline $A, G$, ou $\mathrm{M}$ ni de fraction de $\mathrm{C} 3$ de complément, permettant d'éliminer le diagnostic de pemphigoïde ou de pemphigus. L'examen histologique (fig. 3) montre la présence d'un épithélium irrégulièrement épaissi. On note une orthokératose de surface et un infiltrat lymphocytaire important du chorion sousjacent. Le diagnostic de lichen plan buccal érosif bénin est donc posé.

\section{Cas $n^{\circ} 3$ : Mme 0 .}

Madame O, 32 ans, nous consulte pour des douleurs au brossage et des difficultés d'alimentation qui persistent depuis plusieurs semaines.

Cette patiente, fumeuse (environ 15 paquetsannée), ne nous signale aucun antécédent médical, chirurgical, ni aucune allergie. Lors de l'anamnèse, elle nous fait part uniquement d'un stress important.

L'examen des muqueuses met en évidence des lésions kératosiques de localisations multiples. D'après la patiente, les lésions blanches sont d'abord apparues sur les gencives et sur les bords de langue puis sur la face interne des joues. Les lésions se présentent sous la forme de plages kératosiques avec, en surface, un aspect de réticulations denses et hétérogènes
Suite à cela, la patiente est traitée par des corticoïdes locaux (Buccobet ${ }^{\circledR}$ ), à une posologie de 8 comprimés par jour, pendant 3 mois. Dans le but d'éliminer tout facteur irritant, il est vivement recommandé à la patiente d'arrêter de fumer et des conseils pour améliorer son hygiène bucco-dentaire sont prodigués. Des signes d'activité inflammatoire persistant sur la muqueuse jugale, un traitement par immunosuppresseur topique est proposé avec application de la crème Protopic ${ }^{\circledR}$, une semaine par mois. Puis le traitement par corticoïdes locaux est diminué avec la prise de 4 comprimés de Buccobet ${ }^{\circledR}, 3$ semaines par mois pendant 4 mois de traitement. Une nette amélioration des lésions est enfin constatée. Toutefois, la surveillance bisannuelle du lichen est instaurée.

sur fond de muqueuse érythémateuse, évoquant un lichen de type réticulé.

Au fur et à mesure des différentes consultations, les lésions évoluent et des zones érosives (fig. 4 a et 4 b) sont retrouvées au sein des plages kératosiques, ce qui correspond à des phases symptomatiques de la dermatose (fig. 6 et 7).

$\mathrm{Au}$ vu de ces signes d'activité, des fragments de muqueuse sont prélevés, à cheval sur les zones atteintes et les zones saines périlésionnelles. L'examen histologique confirme le diagnostic de lichen plan buccal érosif (fig. 5).

Un traitement par corticoïdes locaux (Buccobet ${ }^{\circledR}$ ) a été prescrit à raison de 8 comprimés par jour pendant 3 mois. Par ailleurs, la patiente a nettement diminué sa consommation de tabac. Ceci a permis une régression de la symptomatologie. 
À ce jour, après plusieurs années de suivi régulier, les lésions érosives se raréfient et les phases symptomatiques s'espacent. Sur la muqueuse jugale, les lésions kératosiques sont

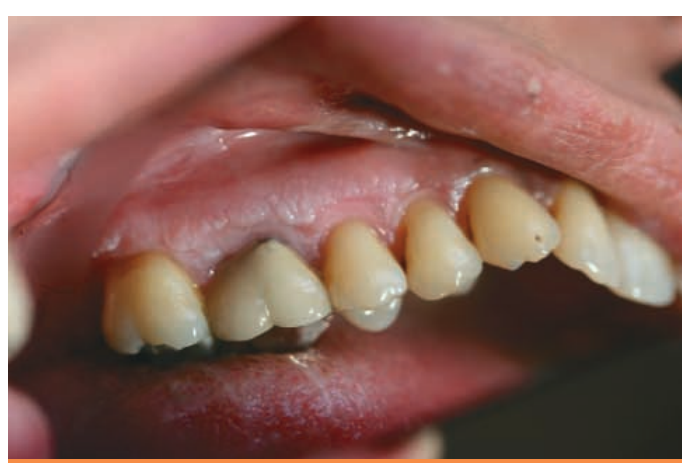

Fig. 4 a Des zones kératosiques sont observées au niveau gingival, avec une zone érosive au collet de la 15 , ici en phase cicatricielle. largement atténuées, occupent une plus petite surface (zone plus inféro-postérieure) et ont pris une forme atrophique qui peut être qualifiée d'état post-lichénien.

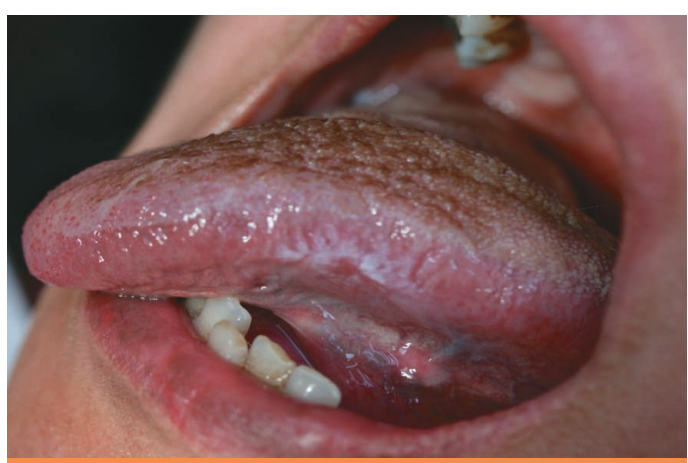

Fig. 4 b On observe une zone kératosique sur le bord latéral gauche de la langue, ainsi qu'une zone érosive au centre de la lésion.

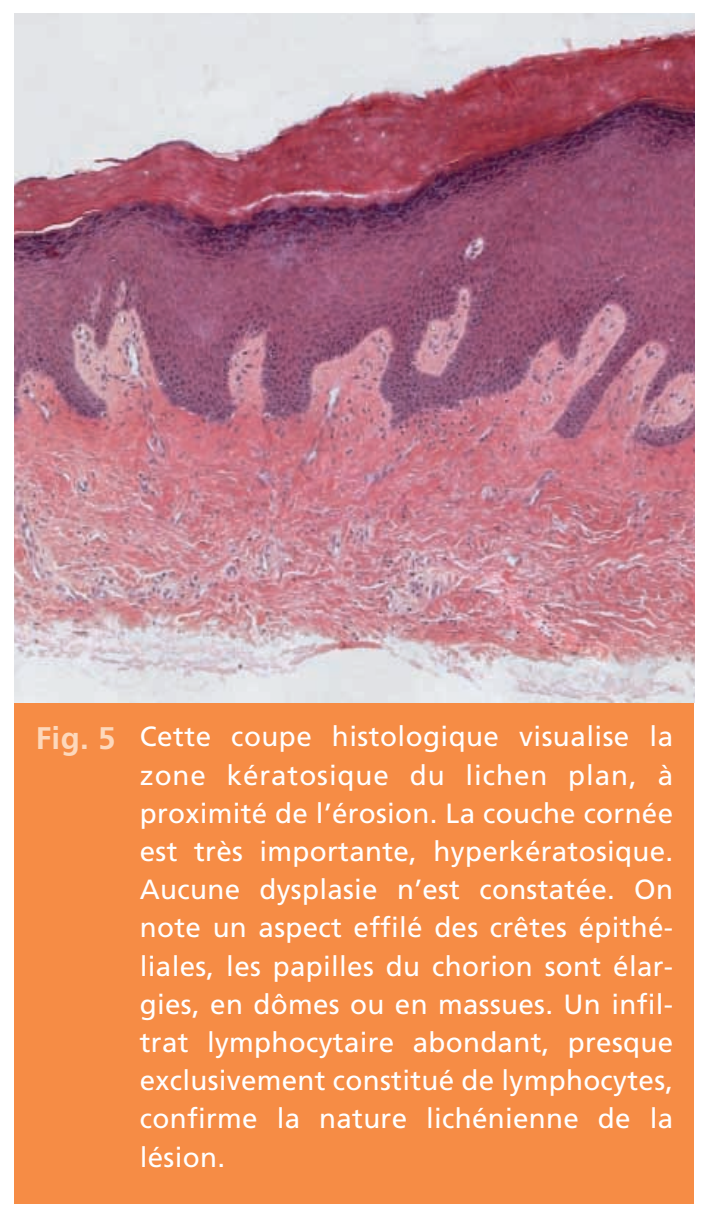




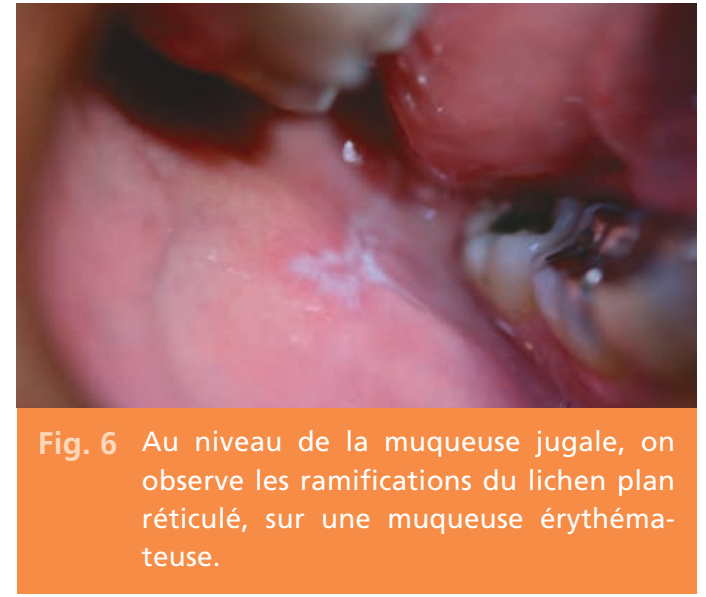

\section{Discussion}

Le syndrome de gingivite érosive chronique a été décrit pour la première fois par Prinz en 1932 sous le nom de gingivite desquamative superficielle, et a été considéré initialement comme une entité associée à des désordres endocriniens [37, 44, 45]. II est maintenant avéré que les gingivites érosives chroniques connaissent des causes aussi diverses que la pemphigoïde bulleuse (39 \% des cas), le lichen plan buccal (36\% des cas), le pemphigus (15\%), plus rarement le psoriasis, la dermatose à lgA linéaire, le lupus ou l'érythème polymorphe post-herpétique [31, 38, 39, 40].

Le diagnostic clinique est particulièrement difficile du fait du caractère très localisé de la pathologie. Dans bien des cas, seule l'immunofluorescence directe permettra de distinguer un lichen plan d'une pemphigoïde, d'un pemphigus ou d'un lupus. Certains signes cliniques, cependant, permettent d'orienter le diagnostic $[41,42,43]$.

Le signe de la pince est un critère d'orientation vers la pemphigoïde cicatricielle et consiste à détacher avec une pince un lambeau d'épithé-

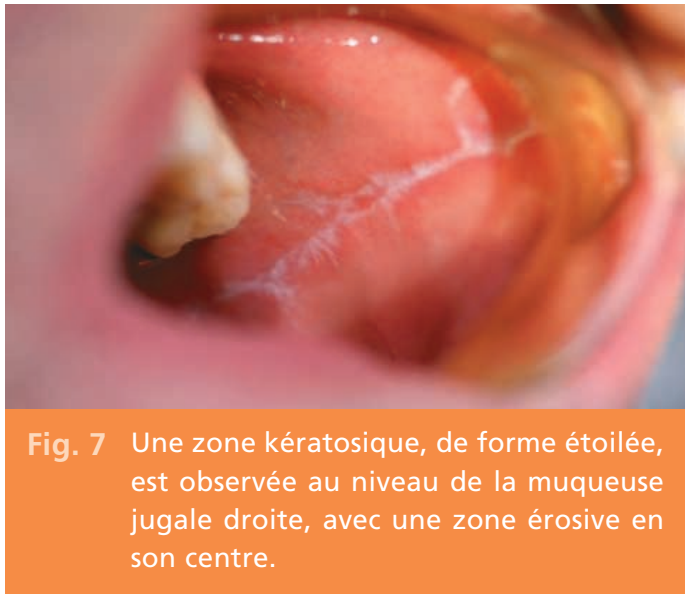

lium en périphérie d'une érosion gingivale ; il est positif lorsque le lambeau mesure plus d'un centimètre [31]. La recherche de lésions associées est également contributive. Ainsi, dans les trois cas présentés, on retrouve en d'autres localisations buccales un aspect typique de lichen plan.

Le lichen plan érosif gingival répond à la corticothérapie. Les corticoïdes topiques sont prescrits en première intention, mais les études ne permettent pas d'établir un protocole clair concernant les formes galéniques, les posologies et les durées d'utilisation [20]. Les corticoïdes systémiques sont également indiqués dans les formes sévères et étendues de LPB résistant à la corticothérapie locale. La prednisone est prescrite à la dose de $1 \mathrm{mg} / \mathrm{kg} / \mathrm{jour}$ pendant une durée de 10 à 15 jours puis une dose dégressive sur un à deux mois [35]. La corticothérapie en bolus, quant à elle, est réservée aux formes diffuses, invalidantes, et en cas d'échec des autres moyens thérapeutiques.

Les rétinoïdes topiques sont utilisés en deuxième ligne thérapeutique, pour leur 
action sur la prolifération et la différenciation kératinocytaire, avec un effet anti-inflammatoire et immunomodulateur. En application topique, l'isotrétinoïne et la trétinoïne se sont montrées efficaces dans plusieurs études [36].

L'utilisation des inhibiteurs de la calcineurine, comme la ciclosporine $\mathrm{A}$ ou le tacrolimus topique est controversée, le résultat des études récentes étant variable quant à leur efficacité [23, 24, 25].

Enfin, dans le premier cas clinique, le lichen plan gingival est noyé au sein d'un pannel

\section{Conclusion}

Le lichen plan érosif gingival reste une forme spécifique de lichen plan buccal, particulièrement réfractaire au traitement et pour laquelle $[28,32,33,34]$ il existe un risque de transformation maligne. Il est donc nécessaire d'informer le patient quant à la nécessité immunopathologique comprenant une thyroïdite d'Hashimoto, un asthme, une dermatose cutanée non étiquetée, une suspicion d'urticaire et une notion d'allergies. Dans la littérature, l'association du lichen plan avec certaines maladies auto-immunes ou inflammatoires comme le syndrome de Gougerot-Sjögren, la sclérodermie systémique, le lupus érythémateux a déjà été décrite $[1,2]$. Existerait-il une susceptibilité immune particulière commune au lichen plan et à de nombreuses autres pathologies?

d'une prise en charge thérapeutique après la réalisation d'un diagnostic clinique et anatomopathologique. De même, l'évaluation du rapport bénéfice-risque doit rester une priorité dans sa prise en charge.

\section{Bibliographie}

1. Chbicheb S, El Wady W. Lichen plan buccal. EMC Stomatologie 22-050-0-10, 2008.

2. Levy A, Le Cleach L. Lichen plan et dermatoses lichénoïdes. EMC Dermatologie (98-525-A-10) 2005:11p.

3. Lozada-Nur F, Miranda C. Oral lichen Planus: epidemiology, clinical characteristics and associated diseases.
Semin Cutan Med Surg 1997;16:273-7.

4. Eisen D. The evaluation of cutaneous, genital, scalp, nail, oesophageal and ocular involvement in patients with oral lichen planus. Oral Surg Oral Med Oral Pathol Oral Radiol Endod 1999;88:431-6.

5. Sugerman PB, Savage NW. Oral lichen Planus: causes, diagnosis and management. Aust Dent J 2002;47:290-7.

6. Lysitsa S, Abi Najm S, Lombardi T Samson J. Lichen Plan Buccal : histoire naturelle et transformation maligne. Méd Buccale Chir Buccale 2007;13:19-9.

7. Boisnic $S$, Frances $C$, Blanchet MC, Szpirglas H, Charpentier $Y$. Imunohistochemical study of oral lesions 
of lichen planus:

diagnostic

and pathophysiologic

aspects.

Oral Surg Oral Med

Oral Pathol 1990;70:462-5.

8. Kolde G, Wesendahl C, Stein $\mathrm{H}$, Reichart PA.

Oral lichen planus:

diagnostic

immunofluorescence

testing on routine

histological material.

Br J Dermatol

2003;148:374-7.

9. Sugerman PB,

Satterwhite K, Bigby M.

Autotoxic T-cell clones

in lichen planus.

Br J Dermatol

2000;142:449-56.

10. Vallejo MJ, Huerta G,

Cerero R, Seoane JM.

Anxiety and depression

as risk factors

for oral lichen planus.

Dermatology

2001;203:303-7.

11. Issa $Y$, Duxbury AJ,

Macfarlane TV,

Brunton PA.

Oral lichenoid lesions

related to dental

restorative materials.

Br Dent J 2005;198:361-6.

12. Martin MD, Broughton $S$, Drangsholt M.

Oral lichen planus and dental materials: a case-control study. Contact Dermatitis 2003;48:331-6.

13. Ibbotson SH, Speight EL, Macleod RI, Smart ER, Lawrence CM.

The relevance

and effect

of amalgam

replacement in subjects with oral

lichenoid reactions.

$\mathrm{Br} J$ Dermatol

1996;134:420-3.

14. Boisrame-Gastrin $S$, Pascal F, Gluckmann E, Deboise A, Berthou C, Predine-Hug $\mathrm{F}$.

Le lichen dans la réaction du greffon

contre l'hôte.

Med Buccale Chir Buccale 2006;12(1):47-55.

15. Bravetti et al.

Le lichen plan buccal

et l'hépatite $C$ :

à propos de 3 cas.

Med Buccale Chir Buccale 1997;3(2):89-94.

16. Hakkou F, Chbicheb S,

El Wady W.

Manifestations buccales

de l'infection virale $\mathrm{C}$

chronique.

Med Buccale Chir Buccale 2009;15(1):31-35.

17. Agbo-Godeau S, Ragot J-P. Leucoplasies

et kératoses buccales.

Actual Odontostomatol

(Paris) 2004;225:23-40.

18. Vaillant $L$, Huttenberger $B$. Pathologie

de la muqueuse

buccale.

Encycl Med Chir

Dermatologie

12-830-A, 1997:14p.

19. Lundquist EN, Wahlin $Y B$, Bergdahl J.

Psychological Health in patients with genital and ral erosive lichen planus.

J Eur Acad Dernatol Venereol 2006;20:661-6.

20. Al-Hashimi l et al. Oral lichen planus and oral lichenoid lesions: diagnostic

and therapeutic

considerations.

Oral Surg Oral Med

Oral Pathol Oral Radiol

Endod 2007;103(1):

S25.e1-S25.e12.

21. Berbis $P$

Acitrétine.

Ann Dermatol 2001;128:737-45.

22. Ho VC, Conklin RJ. Effect of topical cyclosporinerinse on oral lichen planus.

N Engl J Med 1991;325:435.

23. Lozada-Nur Fl, Sroussi HY. Talicromus powder in Orabase $0.1 \%$ for the treatment of oral lichen planus and oral lichenoid lesions: an open clinical trial. Oral Surg Med Oral Pathol Oral Radiol Endod 2006;102(6):744-9.

24. Lundquist $\mathrm{G}$, Forsgren $\mathrm{H}$, Gajecki M, Emtestam L. Photochemotherapy of oral lichen planus. A controlled study Oral Surg Oral Med Oral Pathol Oral Radiol Endod 1995;79:554-8.

25. Kunte $C$,

Erlenkeuser-Uebelhoer I,

Michelsen S,

Scheerer-Dhungel K, Plewig G.

Treatment

of therapy-resistant erosive oral lichen planus with extracorporeal photopheresis (ECP). J Dtsch Dermatol Ges 2005;3:889-94. 
26. Mignona MD, Lo Russo L, Fedele S.

Gingival involvement of oral lichen planus in series of 700 patients. $\mathrm{J}$ Clin Periodontol 2005;32:1029-33.

27. Cendras J, Bonnetblanc JM. Lichen plan buccal érosif. Ann Dermatol Venereol 2000;136:458-468.

28. Epstein JB, Wan LS, Gorsky M, Zhang L. Oral lichen planus: progress in understanding malignant potential and implications for clinical management. Oral Surg Oral Med Oral Pathol Oral Radiol Endod 2003;96:32-7.

29. Ben SlamaL, Boisnic $S$, Samson J, Vaillant L, Fontes V, Frances C. Lichen Plan buccal. Rev Stomatol Chir Maxillofac 2002;103:297-303.

30. Vaillant $L$, Huttenberger B. Pathologie de la muqueuse buccale. Encycl Med Chir (Elsevier, Paris) Dermatologie 12-830-A, 1997:14 p.

31. Vaillant $\mathrm{L}$ et al. Le syndrome "gingivite érosive chronique» étude rétrospective de 33 cas. Ann Dermatol Venereol 2000;127:381-7.

32. Mignogna MD, Lo Muzio L, Lo Russo L, Fedele S, Ruoppo E, Bucci E. Clinical guidelines in early detection of oral squamous cell carcinoma arising in oral lichen planus: a 5-years experience. Oral Oncol 2001;37:262-7.

33. Bornstein $M M$, Kalas $L$, Lemp S, Altermatt HJ, Rees TD, Buser D. Oral lichen planus and malignant transformation: a retrospective follow-up study of clinical and histopathologic data.

Quintessence Int 2006;37:267-71.

34. Holmstrup P, Thorn JJ, Rindum J, Pindborg JJ. Malignant development of lichen planus affected oral mucosa. J Oral Pathol 1988;17:219-25.

35. Kelett JK, Ead RD.

Treatment

of lichen planus with a short course of oral prednisolone. $\mathrm{Br} J$ Dermatol 1990;123:550-1.

36. Piatelli A. et al. Oral lichen planus treated with 13-cis retinoid acid (isotretinoid): effects on apoptotic process.

Clin Oral Investig 2007;11:283-8.

37. Prinz $\mathrm{H}$.

Chronic diffuse desquamative gingivitis. Dental Cosmos 1932;74:331-3.

38. Meynadier J et al. Urticaire chonique : étude étiologique et thérapeutique de $\mathbf{1 5 0}$ cas.

Ann Dermatol Venereol 2000;80:156-7.

39. Cribier B, Noacco G. Urticaire chronique et infections. Ann Dermatol Venereol 2003;130:1S43-52.

40. Juhlin L.

Recurrent urticaria: clinical investigation of $\mathbf{3 3 0}$ patients. Br J Dermatol 1981;104:369-80.

41. Sonoda $T$, Anan $T$, Ono K, Yanagisawa S. Chronic urticaria associated with dental infection. $\mathrm{Br} J$ Dermatol 2001;145(3):516-18.

42. Unger $\mathrm{AH}$.

Chronic urticaria: association with dental infections. South Med J 1960;53:178-81.

43. Shelley WB. Urtcaria of nine duration cleared following dental extraction. Arch Dermatol 1969;100:324-5.

44. Tanphaichitr K. Chronic urticaria associated with bacterial infection.

A case of dental infection. Cutis 1981;27:653-6.

45. Thyagarajan $\mathrm{K}$. Chronic urticaria due to abscessed teeth roots. Int J Dermatol 1982;21:606. 


\section{SUMMARY}

\section{Oral erosive lichen planus: about 3 cases}

Caroline GERBER,

Cécile LUCAS,

Alexandra SOURDOT,

Stéphane KIRCHNER,

Pierre BRAVETTI

$$
\begin{aligned}
& \text { Keywords } \\
& \text { - lichen planus } \\
& \text { oral } \\
& \text { - erosive }
\end{aligned}
$$

Oral lichen planus is a chronical disease, most often benign and diagnosed by means of rigorous clinical and histopathological analysis.

Even if it is characterized by various clinical forms, erosive oral lichen planus corresponds to $40 \%$ of the lesions.

The diagnostic is based on specific symptoms, clinical and histological features. Commonly painfull, this disease necessitates a treatment based on corticosteroids or topic retinoids.

Three clinical cases come to illustrate our comments and enable us to tackle

\begin{tabular}{|c|c|c|}
\hline Paul Heneu & & \\
\hline $\begin{array}{l}\text { Pourquoi } \\
\text { les vaches } \\
\text { ne peuvent-elles } \\
\text { pas descendre } \\
\text { les esCaliers? } \\
\text { et } 2 \text { ag autres questions } \\
\text { de science anusante. }\end{array}$ & Les & $\begin{array}{l}\text { Ces deux ouvrages connaissent un vrai succès depuis leur parution en } 2007 \text { et } 2008 . \\
\text { Leur mission est d'éclaircir toutes ces énigmes scientifiques qui font le quotidien } \\
\text { et de répondre par là aux questions que tout le monde se pose : pourquoi le ciel } \\
\text { est bleu? pourquoi les bulles sont-elles rondes? qu'est-ce qui nous donne envie } \\
\text { de dormir? pourquoi les pingouins marchent-ils en file indienne? } \\
\text { Ils sont le cadeau idéal pour les accros aux quiz, les fanas de science, les éternels } \\
\text { curieux et pour tous ceux qui s'intéressent de près ou de loin au monde qui les } \\
\text { entoure. } \\
\text { Les illustrations sont d'Al Coutelis, dessinateur à Fluide Glacial, auteur } \\
\text { de nombreuses BD et fondateur du journal Cargo Zone. } \\
\text { Paul Heiney est un célèbre auteur, animateur et journaliste. II est aujourd'hui } \\
\text { animateur de télévision, après avoir été journaliste à la BBC, au Times. }\end{array}$ \\
\hline $\begin{array}{l}\cdot 2007 \cdot 978-2-86883-94 C \\
\cdot 192 \text { pages } \cdot 16 €\end{array}$ & $\begin{array}{r}\cdot 2008 \cdot 978-2-7598-00544 \\
\cdot 224 \text { pages } \cdot 16 €\end{array}$ & $\begin{array}{l}\text { Il est l'auteur de plusieurs livres à succès. } \\
\text { À mettre entre toutes les mains! }\end{array}$ \\
\hline
\end{tabular}
association between oral lichen planus and some systemic diseases as well as the topic of desquamative gingivitis.

\section{Deux livres pour toute la famille !}

\section{SPÉCIAL NOËL : FRAIS DE PORT OFFERTS !}

BON DE COMMANDE à renvoyer à :

EDP Sciences - BP $112-91944$ Les Ulis Cedex A

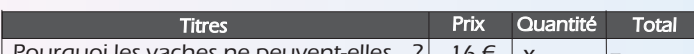

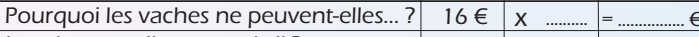

Les chats ont-ils un nombril ?

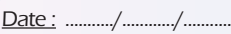

Signature:

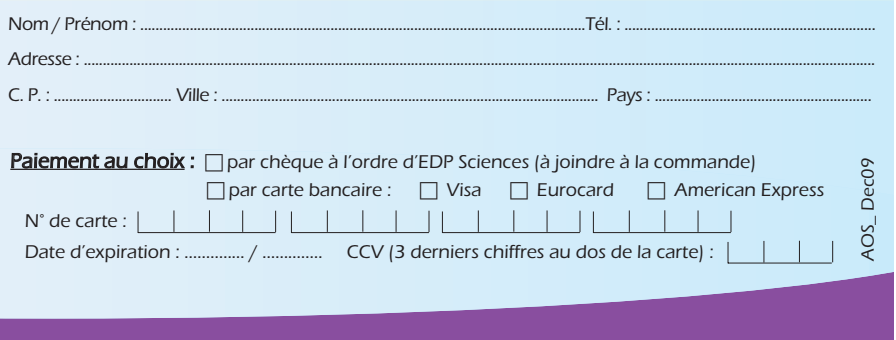

